\title{
POTENCIAL PESQUERO Y ESTUDIOS ECOLOGICOS DE BAHIA MAGDALENA III. LAS EXISTENCIAS DE RAYAS CON ESPECIAL INTERES A LAS YA APROVECHADAS.
}

\section{Por: \\ Christopher Paul Mathews y Jesús Druck González}

\author{
Ciencias Marinas Vol. 2 Núm. I
}

\section{RESUMEN}

Se efeciuó un estudio de las existencias de rayas en la zona de Bahía Magdalena, B. C. Sur, en particular de Dasyatis dipterurus (?). Se notan fluctuaciones estacionales muy marcadas en la abundancia, que serán debidas a movimientos migra* torios. Se presentan datos sobre la estructura de la población de esta especie.

\section{ABSTRACT}

Preliminary results of some stock assesment work which was carried out in the populations of rays in Bahia Magdalena, B. C. Sur are presented, with some special attentions to Dasyatis dipterurus (?). Marked seasonal fluctuations in abundance occur which are probably caused by migratory mevements. Some data on the population of Dasyatis dipterurus ?) are presented.

\section{METODOS}

Se usó la técnica del área barrida para calcular las abundancias de esta especie. Se presentaron detalles sobre el equipo usado y el método de cálculo en otra parte de esta publicación (Mathews y Espinoza). Se asumió una vulnerabilidad de 0.20 por las rayas, puesto que son generalmente grandes y nadan muy rápidamente. Pensamos entonces que el equipo de pesca no podrá capturar las rayas y los otros elasmobranquios con la facilidad que capturaría pescados de escama. Es posible que este valor se aclare a la luz de nuestros estudios futuros; una revisión en la vulnerabilidad tenderá a cambiar los valores de biomasa y de la población calculada. Por esta razón estos valores se presentan en forma muy provisional.

\section{RESULTADOS}

Las capturas totales de rayas en febrero se presentan en la tabla 1. En la última línea se presentan las capturas en agosto de Dasyatus dipterurus, aunque los datos para las otras especies tomadas en agosto no han sido procesados, serán por lo menos igualmente abun- dantes como fueron en febrero. Se destaca que existen poblaciones significativas de rayas en Bahía Magdalena.

\section{TABLA 1}

\section{Captura de rayas en Macapule I.}

Dasyatis dipferurus (febrero) 389 Otros (febrerol 292

Dasyatis dipterurus (agosto) 844

En la figura 1 presentamos las distribuciones geográficas en febrero y en agosto. Se nota que en verano la distribución en Bahía Magdalena es mucho más extensa que en invierno, mientras no ha habido un cambio muy notable en la distribución en Bahía Almejas. Como se pescaron todas las zonas de 2-3 brazas hasta 24 brazas en el invierno, se concluye que hubo un movimiento de las rayas para que fuera ocupada la zona central de Bahía Magdalena en agosto. Las capturas más elevadas de agosto, se cfectuaron en un tiempo total de arrastre que fue de aproximadamente 0.5 el tiempo total de arrastre efectuado en febrero (más o menos 6,300 minutos de arrastre en febrero y 2,900 minutos en agosto, entonces la captura 


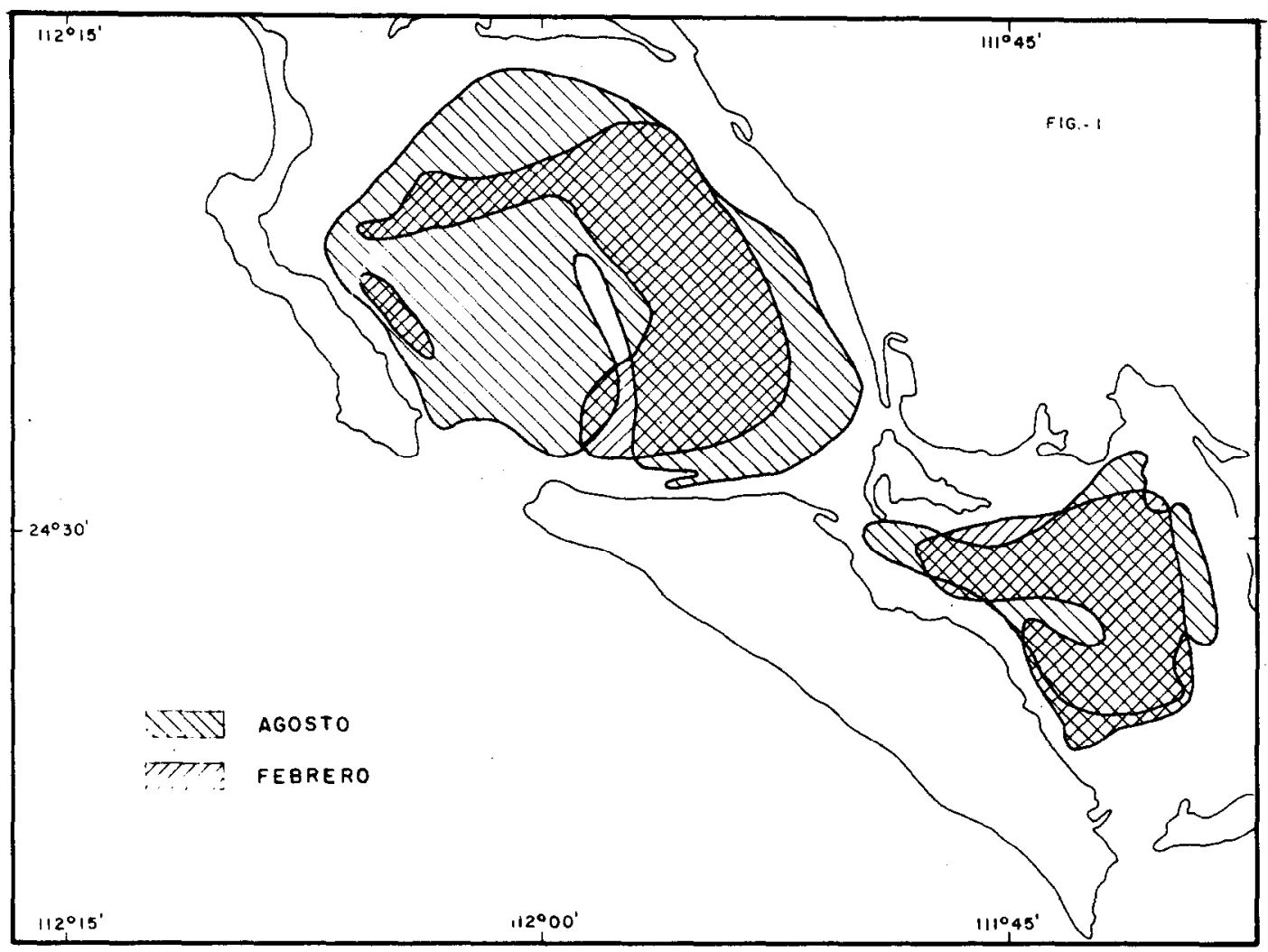

Fig. 1. Distribución de Dasyatis dipterurus en Bahla Magdalena y Bahía Almejas. en febrero y en agasto de 1974.

por unidad de esfuerzo cambió de`3.7 rayas D. dipterurus en 60 minutos de pesca invernal hasta 17.5 D. dipterurus en 60 minutos de pesca veraniega. Esas capturas por unidades de esfuerzo nos sirven como indices aproximados de la abundancia e indican que la extensićn horizontal de las poblaciones de $D$. dipterurus se acompañó con un incremento en abundancia promedio de $x$ 4.7. Estos hechos se explican únicamente por un movimiento de los organismos de aguas de altamar hacia las aguas de la Bahía entre febrero y agosto. En las figuras 2 - 5 se presentan las distribuciones batimétricas de las hembras y de los machos, en los dos cruceros y en las dos Bahías por capturas diurnas y nocturnas. De estas figuras se ciestacan las siguientes conclusiones: (i) que en febrero las rayas hembras abundan mós en Bahía Almejas que en Bahía Magdalena, mientras que en agosto, abundan más, los dos sexos en Bahía Magdalena. En un principio parece que este cambio en la abundancia relativa se deba a que un número mayor de rayas hayan entrado a Bahía Magdalena (ii) las rayas se encuentran en números mucho más elevados a profundidades de 2-13 brazas que de 14-24 brazas en Bahía Magdalena. (iii) que parecen abundar las rayas de los dos sexos más de día que de noche. Esto se deberá probablemente a un cambio de vulnerabilidad de las rayas al equipo de pesca. Posiblemente son las rayas nocturnas; así descansarán en el fondo de día y se escaparían menos fácilmente de día que de noche, cuando su ritmo de actividad sea mayor. Por esta razón, pensamos que las abunancias diurnas sean más precisas. (iv) En todas las profundidades hubieron estaciones sin captura de D. dipferurus; éstas se han presentado en las gráficas. El número de estaciones sin rayas en agosto fue bajo, mientras que en febrero fue muy elevado.

En la tabla 2 se presentan los datos las abundancias promedio de las rayas, 


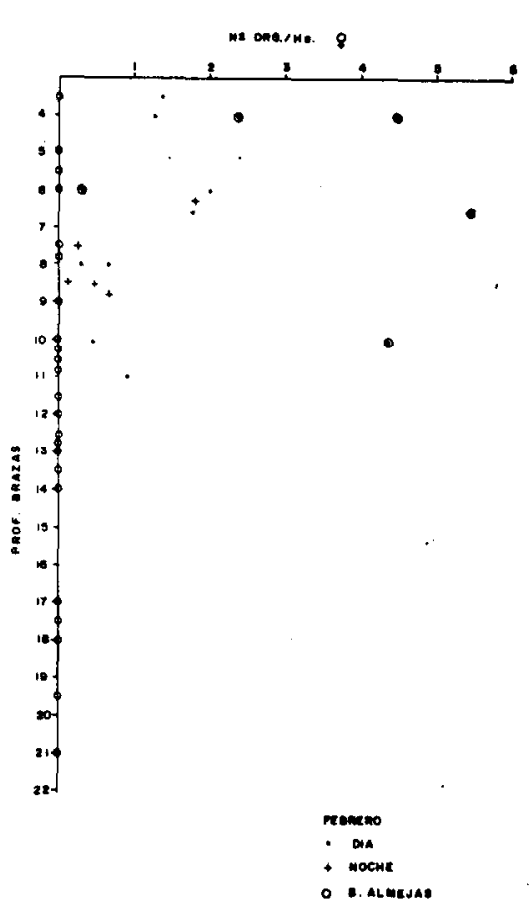

Fig. 2. Distribución balimétrica de las abundancias de Dasyatis dipterurus en febrero 1974 para hembras. Puntos con éricuios representan datos de Bahía Almejas.

las óreas ocupadas por las existencias y el tamaño de las poblaciones invernales $y$ de verano.

\section{TABLA 2}

\begin{tabular}{|c|c|c|}
\hline Abundancia & $\begin{array}{c}\text { Area ocupada } \\
\text { has. }\end{array}$ & $\begin{array}{c}\text { Población } \\
\text { Aprox. }\end{array}$ \\
\hline $\begin{array}{cc}\text { m. he. } \\
1.92 & 1.61\end{array}$ & B. Al. B. Mag. & 112,000 \\
\hline $\begin{array}{c}3.53 \\
\text { AGOSTO }\end{array}$ & 32,000 & \\
\hline $\begin{array}{c}3.36 \quad 3.05 \\
6.41\end{array}$ & 59,000 & 378,000 \\
\hline
\end{tabular}

De esta tabla se destaca que hubo un cambio muy brusco.

Para facilitar el cálculo hemos pasado por alto las diferencias entre las abundancias de Bahía Almejas y Bahía Magdalena. También hemos calculado las abundancias promedios para todas las zonas batimétricas, a pesar de la marcada diferencia entre las abundancias arriba y abajo de las 13 brazas.

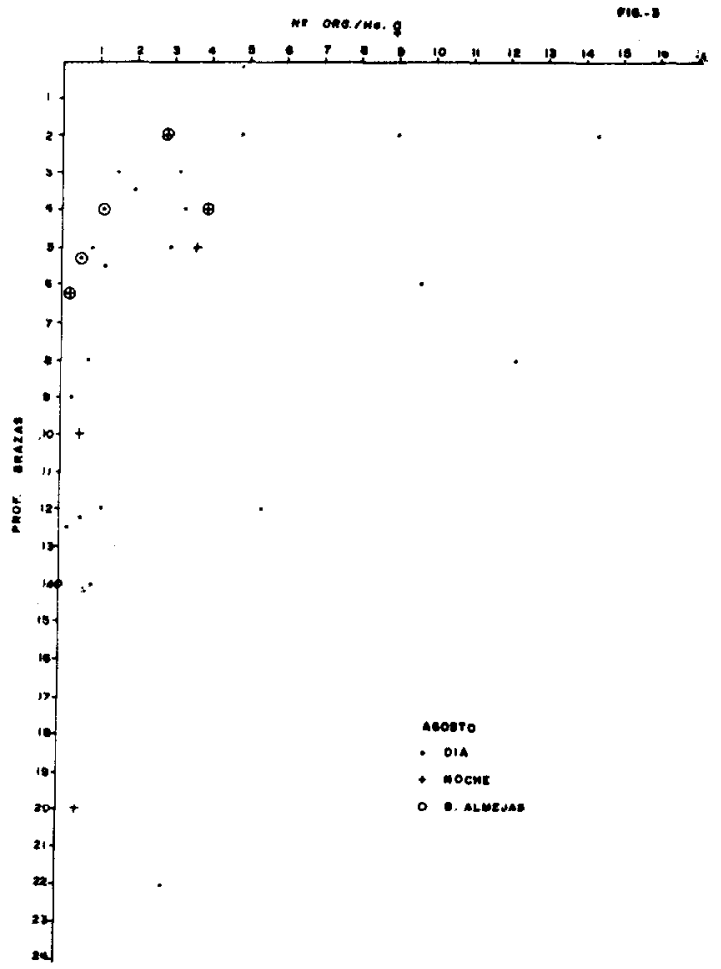

Fig. 3. Distribucián batimétrica de las abundancias de Dasyatis diplenurus para hembras en agosto 1974. Puntos con clrculos representan datos de Bahía Almejas.

Como las aguas mayores a 13 brazas ocupadas por las rayas no tienen área muy elevada, esta simplificación no introduciró un error demasiado grande. Se espera que lleve a una sobreestimación del tamaño total de la población; estos errores se eliminarán cuando se lleve el análisis más adelante, al mismo tiempo las abundancias promedio se calcularon sin tener en cuenta las diferencias diurnas y nocturnas. Esto indudablemente introducirł un error que tiende a subestimar la población. Por ser los errores en alguna medida compensatoria, no pensamo's que el error total sea grave.

En las figuras 6 y 7 presentamos las gróficas de tallas $y$ frecuencias para los machos y las hembras capturados en febrero $y$ en agosto. De estas figuras se destacan las siguientes conclusiones: (i) que los machos dominaron las capturas en las dos épocas de muestreo; (ii) que la mayoría de los machos llegan hasta $60 \mathrm{~cm}$ y que muy pocos llegan a crecer a tamaños mayores, pero que una proporción significativa aunque baja llega 


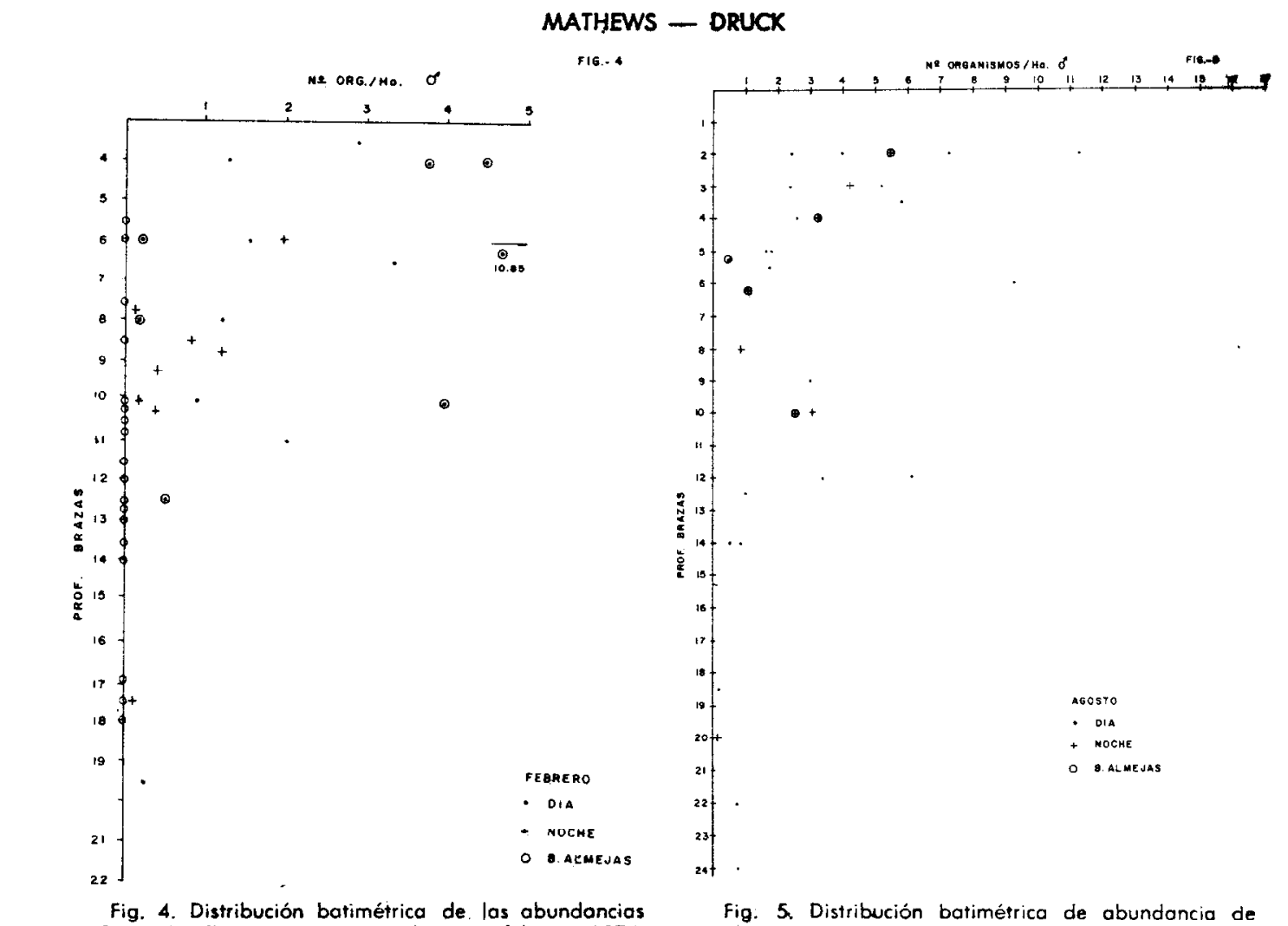

Fig. 4. Distribución batimétrica de las abundancias de Dosyatis dipterurus para machos en tebrero 1974 Puntos con círculos representan datos de Bahía Mogdalena

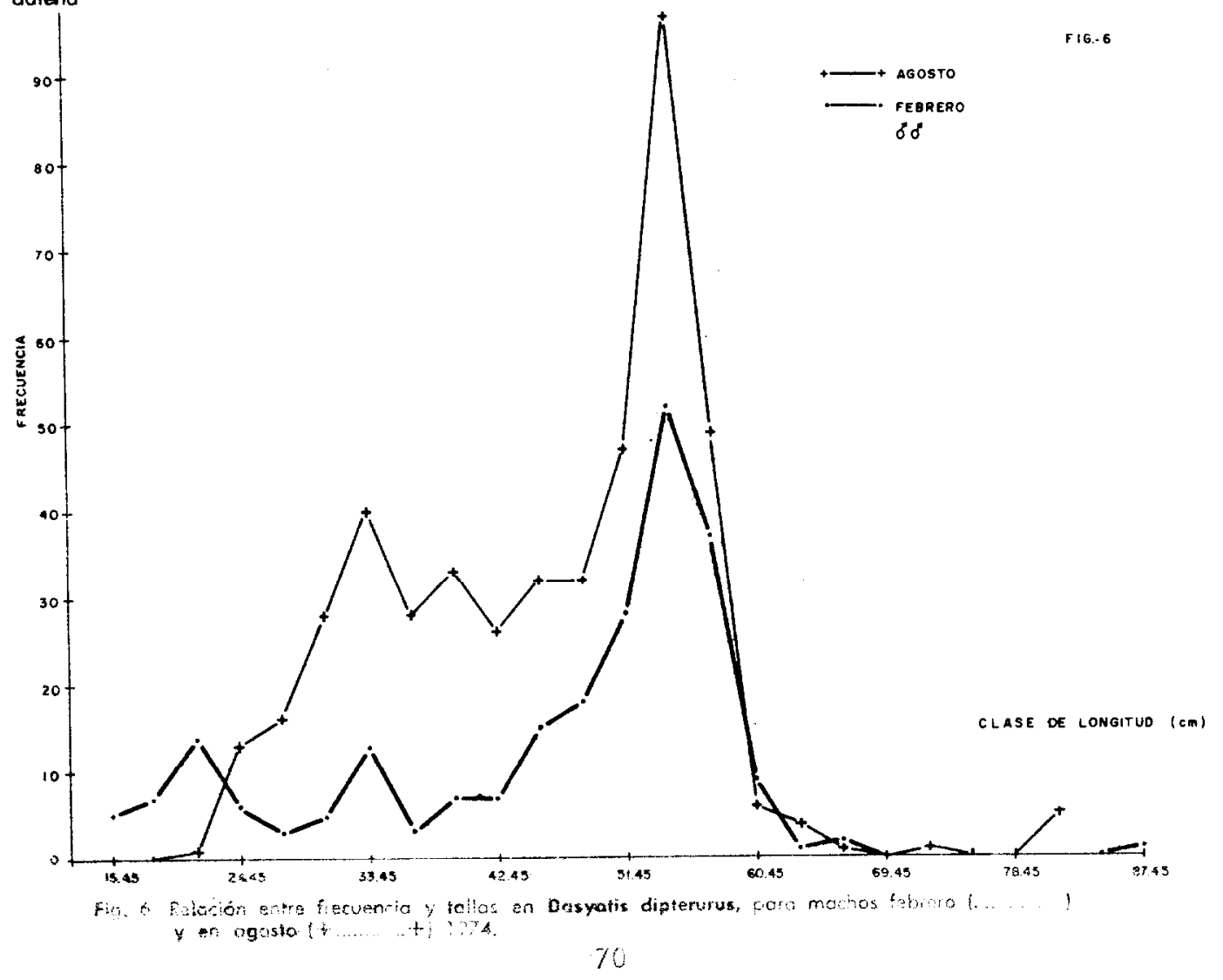

Fig. 5. Distribución batimétrica de abundancia de Dasyatis dipterurus, para machos en agosto 1974. Puntos can circulos representan datos de Bahía Almejas. 


\section{EXISTENCIAS DE RAYAS}

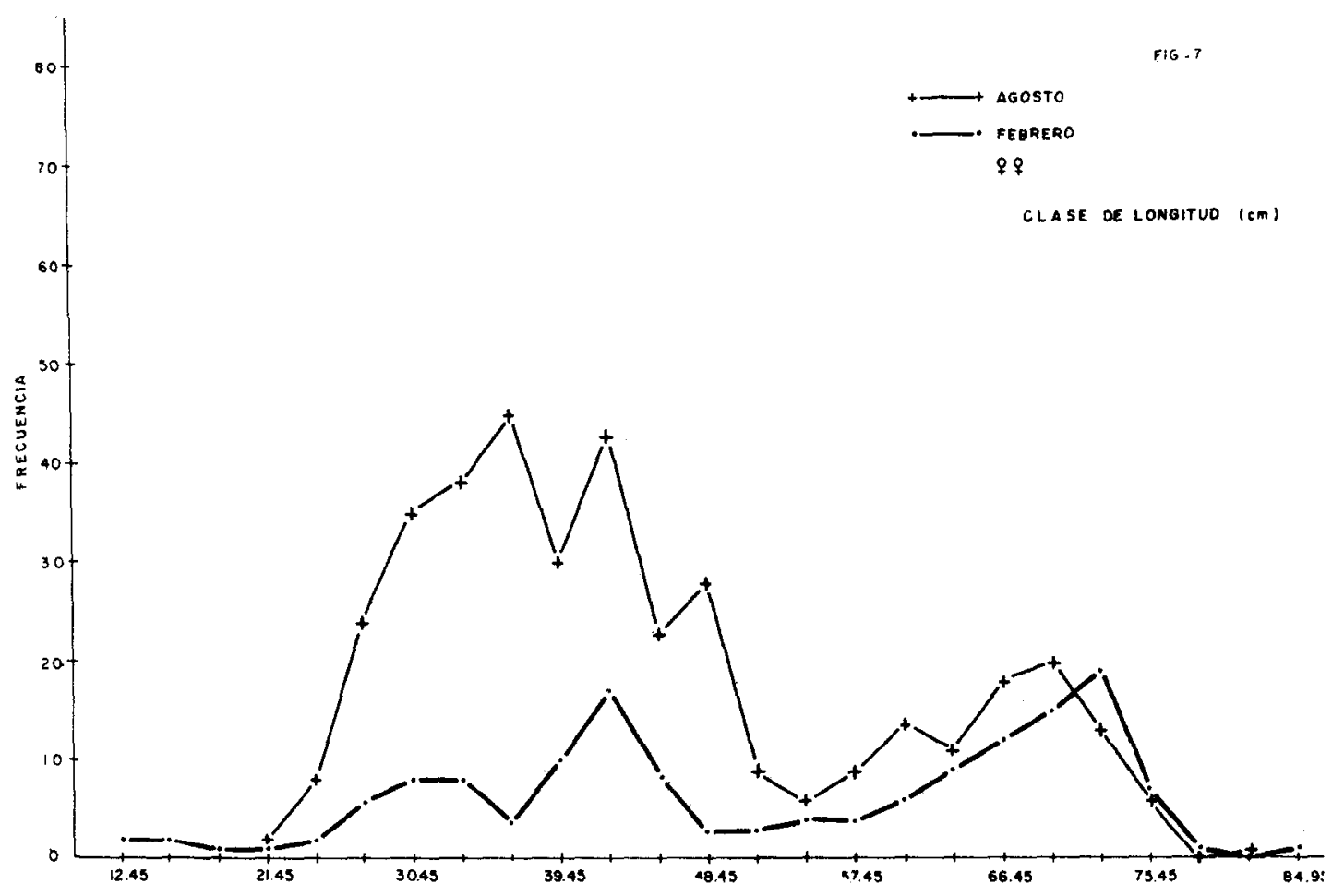

Fig. 7. Relación entre frecuencia y rallas en Dasyatis dipterurus, para hembros en febrero (.............) y en ogosio $1+\ldots \ldots \ldots . .+1197.4$.

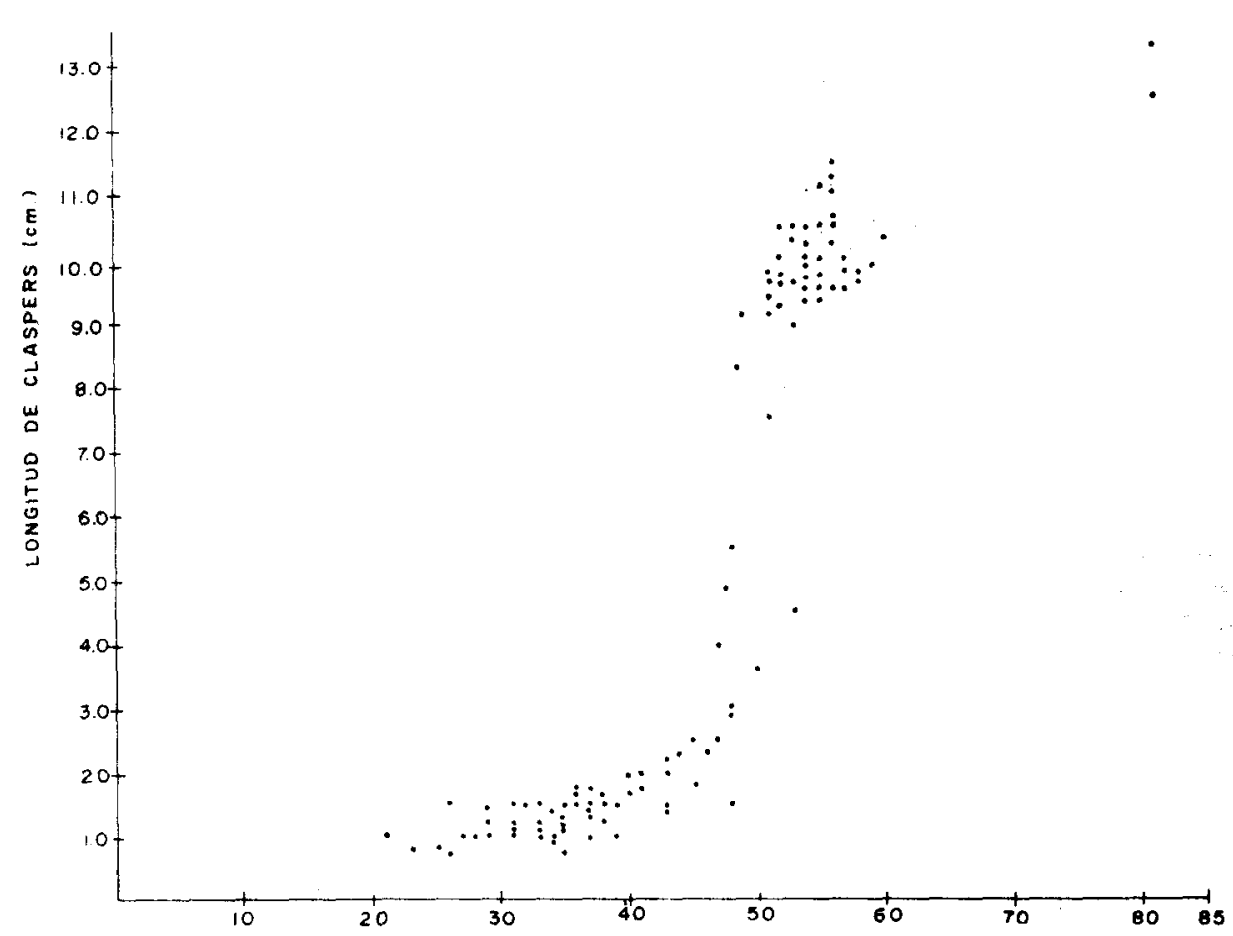

Fig. 8. Relación entre longitud de "claspers" de los machos $\vee$ su longitud. 
(1) -

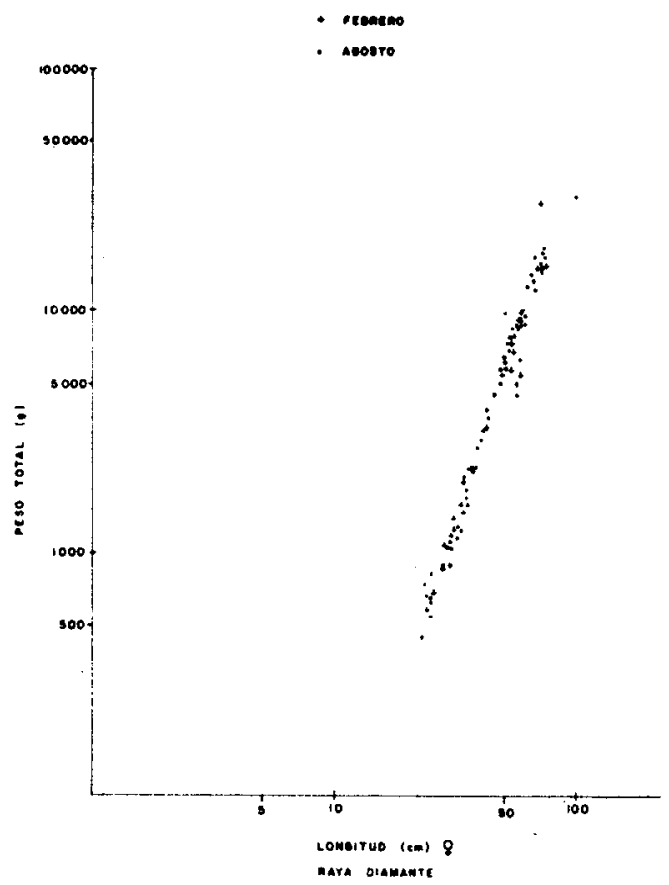

Fig. 9. Relación pesos total y longitud en Dasyotis dipterurus en febrero $(+1$ y en agosto $\{\}$.

hasta los tamaños de $80-90 \mathrm{~cm}$. (iii) Hubieron menos hembras en las capturas de $50-60 \mathrm{~cm}$ que en las clases de tamaño mayor y menor; esto se debe a la presencia de los machos cuyo crecimiento casi cesa en este tamaño mientras que la mayoría de las hembras continúan su crecimiento; (iv) se han excluido de las figuras las hembras más grandes. Individuos raros, de hasta $120 \mathrm{~cm}$ han sido capturados. Las hembras llegan a tamaño mucho más grande que los machos. (v) el aumento de abundancia en agosto se debe a un incremento en el número de rayas pequeñas de $30-60 \mathrm{~cm}$ que es particularmente grande para los de 30-40 $\mathrm{cm}$; la proporción de éstas en agosto es mayor que la de febrero.

Se midieron los claspers de $106 \mathrm{ma}-$ chos a bordo del barco y en la Fig. 8 se presenta la gráfica de longitud del claspers contra longitud total. Se destaca que el tamaño de maduración es de aproximadamente $50 \mathrm{~cm}$ y que todos los individuos mayores de $53 \mathrm{~cm}$ fueron maduros mientras que todos los individuos menores de $47 \mathrm{~cm}$ fueron inmaduros.
En las Figs. 9 y 10 presentamos las gróficas del peso total y longitud total. Las rayas que pudimos pesar llegaron a $30,000 \mathrm{~g}$ pero éste no es el peso máximo hasta el cual llegan. No se puede proporcionar en este momento una cifra precisa para la biomasa de las rayas, ni para el rendimiento cosechable, pero se espera que lleguemos a estas metas en un tiempo no muy lejano.

\section{AGRADECIMIENTOS}

El presente trabajo fue presentado como ponencia en el $V$ Congreso Nacional de Oceanografía celebrado en Guaymas, Son., en octubre de 1974. Durante los dos cruceros que realizó el buque camaronero Macapule 1 en Bahía Magdalena, B.C.S., del 7 al 20 de febrero y del 20 al 27 de agosto de 1974, se capturaron números elevados de rayas. Estas pertenecen a varias especies y géneros, pero fueron suficientemente abundantes para merecer estudio detallado. Hemos escogido la especie Dasyatis diprerurus para darle atención especial en este trabajo debido a que fue la de mayor abundancia y tamañó (hasta $120 \mathrm{~cm}$. de aleta a aleta) y por estar siendo ya aprovechada en forma de una pesquería artesanal muy pequeña.

Quiero agradecer la cooperación de los pescadores de la Cooperativa "Isla Macapule" de Guasave, Sin. y al Capitán Miguel Evaristo Cárdenas del barco Macapule I. También agradecemos la cooperación de la Secretaría de Recursos Hidráulicos con quien se efectuaron estos trabajos bajo contrato can la Dirección de Acuacultura. También agradecemos la ayuda que nos prestó el P.O. Javier Amador Buenrostro Residente de la Dirección de Acuacultura en La Paz, B. C. Sur, y al lng. Sonín Pérez Pérez Director de la Escuela Tecnológica Pesquera de San Carlos B. C. Sur y al Ocean. José Pérez Higuera Sub-Director de la misma. También queremos reconocer la coopeación y ayuda del Biol. Manuel Flores Villegas Jefe de Pesca en Baja California Sur; al comandante del Sector Naval de Puerto Cortés, B. C. Sur Vicealmirante Argudín, y a todos los otros oficiales del Gobierno Federal que nos facilitaron los trabajos en esas zonas. 